\section{Aspectos \\ por considerar \\ para una efectiva \\ integración \\ universitaria \\ mediante \\ las nuevas \\ tecnologías. \\ Una perspectiva \\ desde la \\ sociología de las \\ organizaciones}

\author{
Aleska Cordero*
}

\section{RESUMEN}

En el presente artículo se desarrollan algunas ideas y consideraciones por tomar en cuenta, desde el enfoque organizacional, para fundamentar una efectiva integración iberoamericana entre las instituciones universitarias mediante el uso de las nuevas tecnologías. Se presentan ciertos conceptos prove-

\footnotetext{
* Docente, instructora de la Universidad Nacional Abierta de Venezuela desde 1989. Socióloga, especialista en Desarrollo Organizacional. Actualmente es editora de la Revista Informe de Investigaciones Educativas de la Dirección de Investigación y Posgrado de la UNA, en Caracas.
}

nientes de la teoría organizacional (cultura organizacional, cultura profesional, resistencia al cambio) mediante los cuales es posible abordar el análisis de los problemas que se plantean en las instituciones a distancia en el momento de implantar nuevas tecnologías, intercambiar recursos humanos especializados, adelantar acuerdos de cooperación internacional, etc.

\section{NTRODUCCIÓN}

La integración Iberoamericana es un ideal de vieja data que con la llegada del nuevo milenio se hace más imprescindible y más factible que nunca.

Imprescindible porque ante los evidentes procesos de la globalización, se hace necesario buscar los puntos comunes (que son muchos) entre los países de Iberoamérica para conformar un bloque que consolide y preserve nuestra identidad particular. Factible, porque los avances en las áreas de la comunicación y de la informática realmente potencian, facilitan y agilizan los contactos e intercambios entre las instituciones de educación superior de nuestros países. Sin embargo, estos avances en tecnología son herramientas que, para coadyuvar efectivamente al proceso de integración, necesitan de una plataforma cultural, social 
y organizacional que será el tema de este artículo. En efecto, no basta con poseer las tecnologías más avanzadas en comunicación e informática para establecer un dialogo certero entre nuestras instituciones y países, se deben revisar algunos aspectos como base para la acción, aspectos que pueden obstaculizar o potenciar este intercambio en el contexto de las instituciones de educación a distancia.

\section{Integrar respetando y entendiendo la diversidad}

¿Qué significa Integrar? Según el Diccionario de la Lengua Española de Kapelusz, integrar es "hacer un todo con partes distintas" (Kapelusz, 1979:864). Las bases para la integración son a nuestro entender, y en el contexto planteado en este trabajo, el conocimiento mutuo y la cooperación en función de la identificación de puntos comunes, respetando la diversidad y las diferencias entre los países y las instituciones, en sus modos particulares de ver y hacer las cosas.

La afirmación anterior implica la consideración de dos premisas:

a) La primera, referida a los procesos de globalización que, queramos o no, constituyen una tendencia mundial en la cual están inmersas las universidades iberoamericanas. Como señala Casas (2001) esta tendencia "obliga" a la institución universitaria a buscar alianzas estratégicas en distintos niveles si quiere mantenerse vigente frente a la avalancha de cambios que acarrea el desarrollo de las nuevas tecnologías a nivel mundial.

Estas alianzas nacionales o extra-nacionales permiten a las universidades comprometidas en acuerdos e intercambios fortalecerse como organizaciones y ampliar sus posibilidades de llevar a cabo con excelencia las funciones que les son propias (docencia, investigación y extensión).

b) La segunda premisa implica considerar que la globalización tiene también su lado "oscuro" y ante la tendencia a la homogeneización social y cultural que impone, se hace urgente que nuestras instituciones universitarias conozcan a fondo sus particularidades para preservar así aquellas que les otorgan "identidad corporativa" y delimitan su "personalidad organizacional. 
En el presente artículo se desarrollan muy brevemente algunas ideas que apuntan a la existencia de factores organizacionales que merecen tomarse en cuenta como plataforma para la búsqueda de soluciones conjuntas a problemas similares de las instituciones a distancia en nuestros países (necesidad de automatizar los procesos administrativos, mejorar la asesoría y ampliar su radio de acción, disminuir la deserción estudiantil típica en esta modalidad de enseñanza, diseñar nuevas estrategias de aprendizaje, etc.). Para ello se presentan algunos conceptos provenientes de la teoría y práctica organizacional que pueden ayudar a comprender la complejidad organizativa de la institución universitaria y aclarar su panorama para diseñar los mecanismos idóneos de integración interuniversitaria mediante la utilización adecuada y consciente de las nuevas tecnologías.

Se desarrolla entonces brevemente una reflexión en torno a:

a) Las características organizacionales de la universidad como organización social.

b) La definición de prioridades institucionales.

c) La existencia de la resistencia al cambio y la búsqueda del liderazgo idóneo. d) La existencia de subculturas organizacionales.

\section{Las universidades como organizaciones sociales particulares}

Un primer aspecto por considerar para viabilizar la deseable integración entre nuestras instituciones es el hecho de que las mismas son formas de organización social con el objetivo explícito de transmitir conocimiento (función docente), producir nuevos conocimientos (función de investigación) y difundir este conocimiento entre los otros sectores de la sociedad (función de extensión). Estas formas particulares de organización social están sumidas en un contexto social y cultural dinámico y complejo. La teoría de las organizaciones ha venido trabajando algunos conceptos que pueden ser de utilidad para comprender la dinámica tanto interna como externa de este tipo de organizaciones. Queremos destacar tres de estos: cultura organizacional, cultura nacional y cultura profesional. Estos conceptos están entrelazados con otras variables organizacionales como son: la estructura administrativa, el liderazgo y la gerencia de recursos, entre otros, pero de acuerdo con varios autores (Pettigrew, 1979; Shein, 1988; Hofstede, 1991; Denison, 1990 y Clavier y Llopis, 1993, entre otros) la cultura nacional y la cultura orga- 
nizacional conforman por sí mismos marcos teóricos conceptuales generales e integrales que ayudan a comprender y estudiar el comportamiento de los individuos y grupos en las organizaciones. El concepto de cultura nacional trabajado por Hofstede (1991) por ejemplo, indica la programación mental compartida por grupos de individuos pertenecientes a un mismo territorio geográfico. Esta programación se refiere a patrones de conducta, valores, creencias y actitudes que son comunes a los individuos de una nación y que generan una visión del mundo y de las relaciones sociales propias de esa nación. Algunas investigaciones demuestran (Toro, 1993; Laurent, 1991; Granell, 1997 y Hofstede, 1991) la poderosa influencia de la cultura nacional en las estrategias gerenciales y de negociación de los empresarios de diferentes países.

El otro concepto útil es el de cultura organizacional. Este se refiere (Etkin y Schvarstein, 1997:201) a "los modos de pensar, creer y hacer cosas en un sistema, se encuentren o no formalizados. Estos modos sociales de acción están establecidos y son aplicados por los participantes mientras pertenecen a los grupos de trabajo, incluyendo formas de interacción comunicativa transmitidas y mantenidas en el grupo, tales como lenguajes propios del sistema, liderazgos internos o pre- ferencias compartidas". La cultura organizacional puede asumir características diversas según sea el tipo de organización (en este caso, las universidades), su forma de relación con el Estado (públicas o privadas), el tipo de estructura organizacional adoptada, etc. Como lo señalan Clavier y Llopis (1993:91) la cultura organizacional (o corporativa) es tan poderosa que puede hacer fracasar o desviar cualquier estrategia gerencial no acorde con ella o que no la tome en cuenta. En la realidad de esta cultura organizacional es donde los estudiosos han encontrado la causa principal del fracaso de muchas estrategias gerenciales y administrativas, transferidas sin análisis previo ni adaptaciones, desde contextos ajenos a la organización en la cual se pretende implantarlas. Lo mismo sucede cuando se transfieren o introducen tecnologías sin consideraciones como las planteadas (Silvio, 1991).

La intersección de ambos conceptos, cultura nacional y cultura organizacional, constituye a nuestro modo de ver una herramienta útil para analizar y comprender la dinámica particular de cada organización, los elementos comunes que se derivan de la modalidad educativa compartida (la educación superior a distancia) y, fundamentalmente, para analizar qué modelos, elementos y aspectos de 
las nuevas tecnologías pueden adaptarse mejor y con menos dificultades a nuestra cultura institucional particular.

\section{Definición de prioridades de acuerdo \\ con las capacidades reales de una institución}

La discusión sobre este punto surge de la preocupación al observar que muchos acuerdos de integración, es decir de intercambio de recursos y trabajo cooperativo potencial entre las instituciones universitarias, quedan sin efecto al no trascender el mero compromiso formal, y tener una existencia limitada al papel. Independientemente de la voluntad que puede dar origen a estos potenciales intercambios es menester tomar en consideración a la hora de establecer acuerdos o adoptar nuevas tecnologías, lo que en literatura gerencial se conoce como el cuadrante de las oportunidades y ventajas de las organizaciones (Kotter, 1990). La identificación de las fortalezas y debilidades de cada institución es una función de la alta gerencia que sirve de marco para la definición de las estrategias de cooperación interuniversitaria en las cuales pueden inscribirse los acuerdos de integración. La identificación y definición de los recursos con que cuentan nuestras universidades a distancia permite, entre otros objetivos la difusión de experiencias valiosas originadas en una determinada universidad, pero transferibles a otros contextos buscando resolver problemas comunes aunque no idénticos que afecten y son propios de la modalidad a distancia (formas alternativas de acreditación de los aprendizajes, la evaluación y su confiabilidad, el diseño de textos de calidad y su efectiva distribución, etc.). Saber qué tiene cada institución, en qué áreas y disciplinas posee mejor capacidad y fortaleza, qué áreas o recursos es necesario fortalecer de acuerdo con las prioridades de cada una y en resumen, qué puede ofrecer o tomar ventajosamente de otras instituciones a distancia, constituye una base fundamental para potenciar efectivamente la integración a la que aludimos.

\section{La resistencia al cambio y el liderazgo necesario para romper barreras entre nuestras instituciones}

El fenómeno de la resistencia al cambio ha sido un aspecto muy estudiado dentro de las organizaciones. La introducción de innovaciones tecnológicas no siempre es bienvenida dentro de nuestras universidades por diversas razones, entre ellas porque las organizaciones son demográficamente hetero- 
géneas, es decir, sus integrantes poseen diferentes niveles educativos, económicos, etarios, etc. La conformación de la estructura organizacional sumada a esta particular estructura demográfica afectan de diferente forma la necesidad que tiene cada institución de adoptar innovaciones y establecer intercambios con "iguales". El conocimiento y efectiva consideración de la cultura organizacional y de los distintos sectores demográficos diferenciables dentro de las universidades pueden señalar qué nivel de resistencia al cambio puede existir a la hora de adoptar nuevas tecnologías para la integración. Esta resistencia al cambio puede "sabotear" las mejores intenciones. Es en ese punto en donde se hace útil la identificación de agentes motivadores en cada institución, que lideren los procesos de cambios actitudinales necesarios para congregar a los equipos de trabajo en torno a un propósito predeterminado sea este de integración interinstitucional o simplemente de introducción de nuevas tecnologías en el ambiente laboral y que diseñen, sobre todo, las estrategias adecuadas a un contexto nacional, institucional o internacional según se requiera.

\section{La existencia}

de subculturas

o cómo gerenciar

las diferencias internas y transnacionales

El tercer concepto útil para comprender la dinámica de nuestras universidades y vinculado a lo planteado anteriormente es el de cultura profesional o cultura ocupacional (Van Maanen, 1984). En efecto, en las universidades se congregan estamentos ocupacionales de diversa índole; una primera clasificación nos remite a la diferenciación entre personal académico y personal administrativo. Ambas subculturas poseen diferente manera de "entender" su lugar en la institución (Picón, 1994). Por ejemplo, en el caso de las instituciones a distancia, la perspectiva de los "asesores académicos locales" o personal docente que trabaja en contacto directo con el estudiante en los centros regionales es seguramente diferente a la que tienen los docentes de los niveles centrales de la misma institución. La accesibilidad a las nuevas tecnologías comunicacionales por parte del personal universitario variará también de acuerdo con el país, con las posibilidades financieras de cada institución, con las prioridades que se le asigne a la actualización tecnológica en la institución y con el nivel de "alfabetismo 


\section{ESPUGA}

informático" (Casas, 1996:15) o cultura tecnológica de cada sector de la organización. Por ende, debe hacerse consciente para la alta gerencia a la hora de establecer bases para los acuerdos de cooperación interuniversitaria, que los mecanismos para la integración en proyectos que involucren al personal de cada institución y a los subalternos que convivan en ella necesitan un ritmo y unos requerimientos diferentes. La intersección entre culturas nacionales y organizacionales también podría convertirse en interesante problema de investigación conjunta entre nuestros países. Para finalizar, tomemos en cuenta lo que señala Lolas (1998:80): “Las nuevas tecnologías están produciendo una avalancha inédita de información". Sin embargo, "la accesibilidad a esa información no es un atributo de la información misma sino de las personas e instituciones que la producen, manejan y transmiten". La responsabilidad de la estructuración y uso de la información sigue estando en manos del individuo, y de la organización y las nuevas tecnologías que solo le permiten potenciar la velocidad y ampliar la forma de su distribución. Si bien las universidades pueden adquirir y aprender a usar eficientemente las nuevas tecnologías, la calidad de lo que trasmiten a sus estudiantes y a otros co- legas seguirá siendo un atributo de su capacidad interna e integrada de producir el conocimiento y compartirlo (Contasti, 1990), independientemente del "parque tecnológico" que poseen. En consecuencia, la integración de las universidades a distancia de Iberoamérica debe en principio pasar por la detección de problemas comunes a todos los sistemas y en la proposición de soluciones también comunes, más no idénticas, de acuerdo con una agenda de prioridades que solo pueden establecer las propias instituciones involucradas. Los altos índices de deserción de los sistemas a distancia, el uso de nuevas tecnologías para el proceso de aprendizaje y otros temas semejantes son problemas comunes y pueden ser estudiados de manera conjunta tomando en cuenta las diferencias demográficas y culturales de instituciones y países que existen y que hemos señalado a lo largo de este trabajo.

\section{CONCLUSION}

Una efectiva integración interunivesitaria e internacional mediante las nuevas tecnologías de la informática y de la comunicación sólo será factible si está basada en la consideración de algunos de los aspectos señalados y que resumimos: 
a) La utilización de las tecnologías informáticas y comunicaciones no son la panacea ni sucedáneos de la integración interuniversitaria iberoamericana. Aun las más avanzadas sólo son herramientas, ya que potencian y agilizan la integración pero no pueden por sí mismas crearla ni hacerla permanente. La calidad y pertinencia de los recursos, conocimientos e información intercambiadas son atributos de las personas e instituciones que los seleccionan, manejan y transmiten, no de las tecnologías que les sirven de canales de comunicación.

b) La introducción y utilización de las nuevas tecnologías informáticas y comunicacionales pueden modificar las estructuras organizacionales y de comunicación de las universidades. Y también afectan a las personas involucradas. Por ende, es menester tomar en cuenta, a la hora de diseñar mecanismos de integración interuniversitaria, la existencia de "modos de hacer las cosas", es decir culturas organizacionales, profesionales y nacionales en cada institución y país. La eficiencia, permanencia y pertinencia de estos mecanismos y redes de integración sólo serán posible si sabemos que: 1) puede presentarse la resistencia al cambio, 2) deben identificarse los factores motivacionales que lideren los proyectos adecuadamente y 3 ) la forma de asumir y entender el intercambio institucional e internacional, puede variar entre los sectores de una misma institución y entre las instituciones de una región tan vasta como la iberoamericana.

c) Las plataformas socioculturales para la integración son, como dijimos, el conocimiento mutuo y la cooperación. Por ende, es útil que previamente cada institución se conozca internamente, es decir mediante un análisis sincero de sus debilidades y fortalezas, para así establecer intercambios sobre la base de un conocimiento ajustado a la realidad en torno a sus recursos efectivos y potenciales. En otras palabras, si somos diferentes, en qué lo somos, sí somos iguales en qué lo somos, sobre qué puntos prioritarios deben establecerse los acuerdos de cooperación, integración e intercambio y sobre cuáles herramientas tecnológicas podemos apoyarnos, sin alterar ni irrumpir abruptamente los procesos humanos dentro de las organizaciones. 


\section{BIBLIOGRAFÍA}

Casas, M. (1996). “Universidades Latinoamericanas a Distancia. Panacea versus realidades". En: Revista Informe de Investigaciones Educativas, Vol. X. $\mathrm{N}^{\mathrm{o}} 1$ y 2 (13-34). Caracas - UNA.

Casas, M. (2001). “Conferencia: Ejemplo de Universidad Virtual en Operación. La Universidad Abierta de Cataluña". Dirección de Investigaciones y Postgrado. UNA. Caracas - Venezuela (marzo).

Clavier y Llopis (1993) “Cultura Corporativa y Estrategia Empresarial. Implicaciones Recíprocas". En: Revista Alta Dirección, Vol. 19 (89-93). España.

Contasti, Max (1990) “Espejismo Tecnológico. Desarrollo. Educación a Distancia" En: Villarroel y Pereira (Editores) La Educación a Distancia: Desarrollo y Apertura. Caracas: Fondo Editorial de la Universidad Nacional Abierta, Open Learning Agency e ICDE (52-54).

Costello, N. (1993) “Organizational Culture and Distance Learning". En: Open Learning. Junio (3-11). Canadá.

Denison (1990) Cultura Corporativa y Productividad Organizacional. Bogotá: Legis.

Etkin, J. y Schvarstein, L. (1997). Identidad de las Organizaciones. Invariancia y Cambio. Buenos Aires: Edit. Paídos. 4ta. reimpresión.

Granell, Elena (1997). Éxito Gerencialy Cultura. Retos y Oportunidades en Venezuela. Caracas: Ediciones del Instituto de Estudios Superiores de Administración (IESA).

Hofstede, G. (1991). Cultures and Organizations: Software of the mind. London: Mc Graw Hill.

Kotter, J. (1990). "What Leader Really do?." En: Harvard Business Review, mayo-junio (103-111). USA.
Laurent, A. (1987). “The Cross - Cultural Puzzle of International Management". Human Resource Management. Vol 25 (1) (91-102).

Lolas, F. (1998) Citado por Gómez, Iglesias y Beltrán "Librun: Un mecanismo para la difusión y comercialización del libro universitario". En: Revista Interamericana de Bibliotecología, enero - junio, vol. 21 \# 1 (78-84).

Pettigrew, A. (1979). “On Studying Organizational Cultures". En: Administrative Science Quarterly, Vol. 24, December (570-581). USA.

Picón, G. (1994). El Proceso de convertirse en Universidad. Aprendizaje Organizacional en la Universidad Venezolana. Caracas: Universidad Pedagógica Experimental Libertador y Universidad Experimental Simón Rodríguez.

Shein, E. (1996). "Culture: The missing Concept in Organization Studies". En: Administrative Science Quarterly, Vol. 41 (229 -240). USA.

Silvio, José (1991). "Gestión de Información y Conocimiento en la Educación Superior en América Latina y el Caribe". En: Oportunidades de Conocimiento y de la Información (Vol. II) Reunión Internacional de Reflexión sobre los nuevos roles de la Educación Superior. Caracas: UNESCO/ CRESALC (111-118).

Toro, Fernando (1993). “Diferencia en el Perfil Motivacional de Gerentes de Empresas Públicas y Privadas". En: Revista Latinoamericana de Psicología. Vol. 25 \#3 (403-423). Bogotá.

Van Maanen, J. y otros. (1984). “Ocupational Communities: Culture and Control in Organizations". En: Research in Organizational Behavior, 6 (287-365) Greenwich JAI Press. 